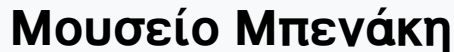

Tóp. 4 (2004)

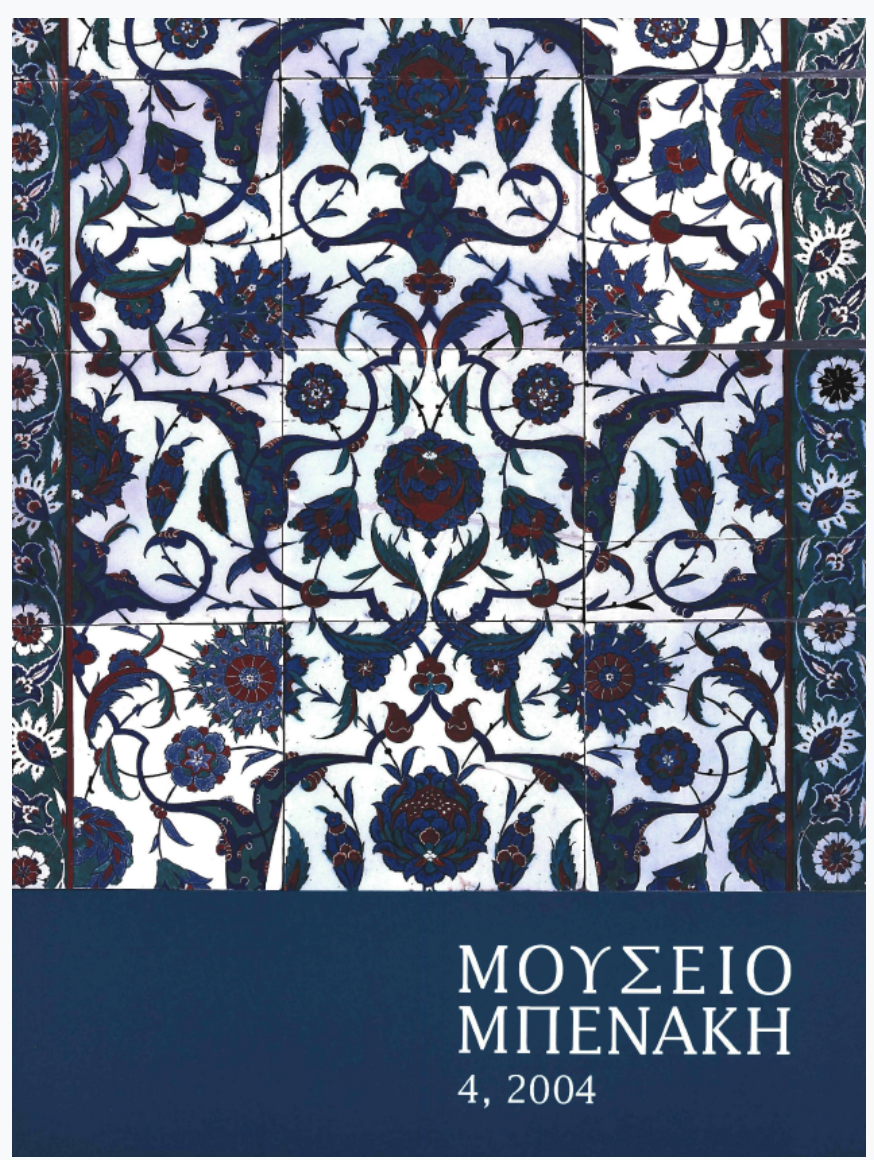

H Revue Britannique kaı $\eta \varepsilon \lambda \lambda \eta v \iota k n ́$

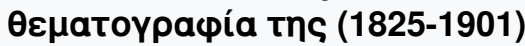

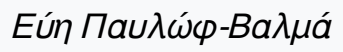

doi: $10.12681 /$ benaki.18256

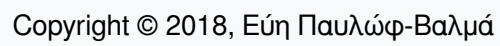

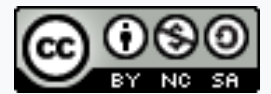

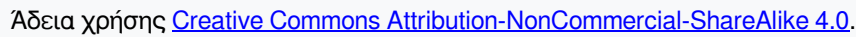

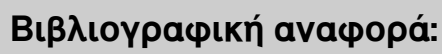

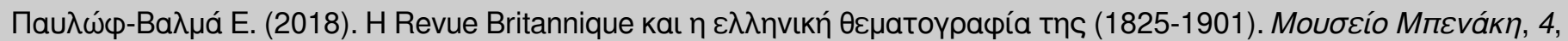
99-105. https://doi.org/10.12681/benaki.18256 


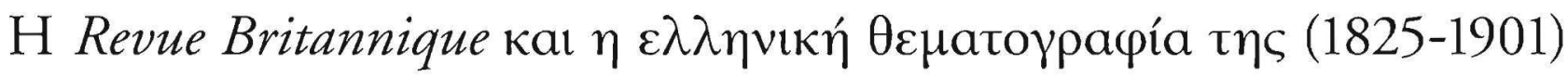

THN ПLAPOY

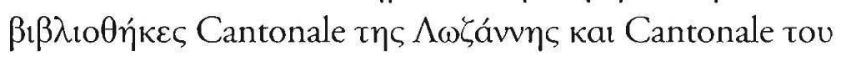

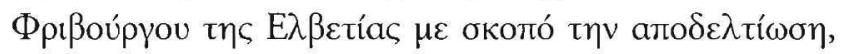

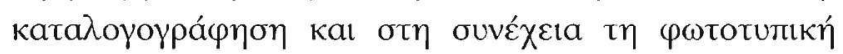

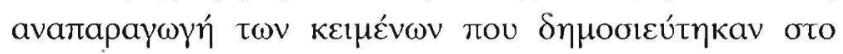

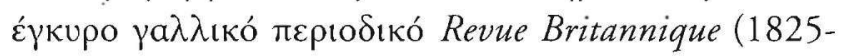

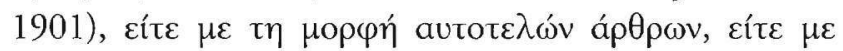

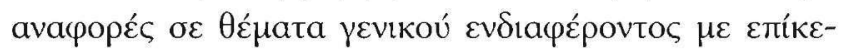

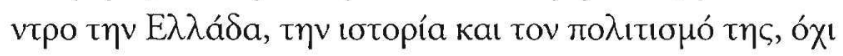

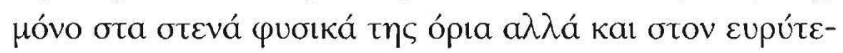

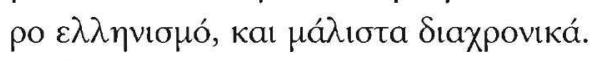

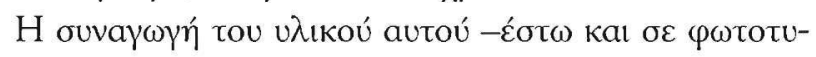

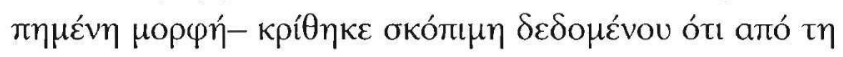

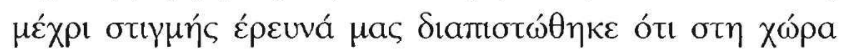

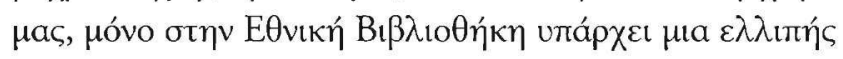

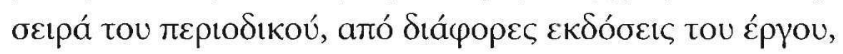

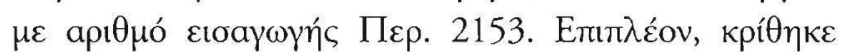

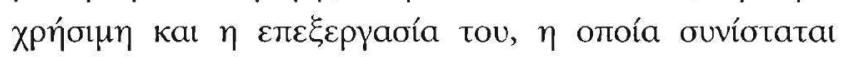

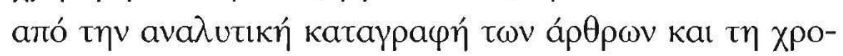

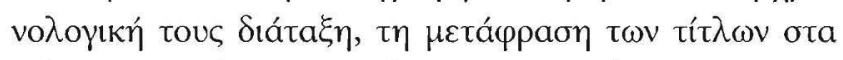

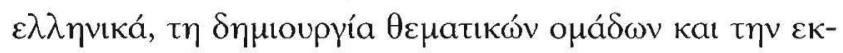

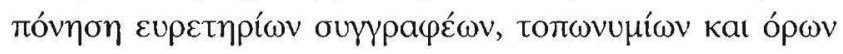

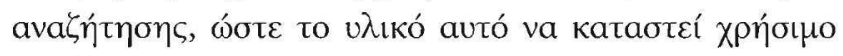

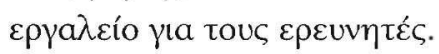

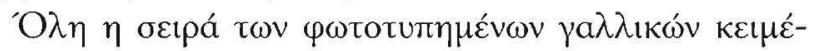

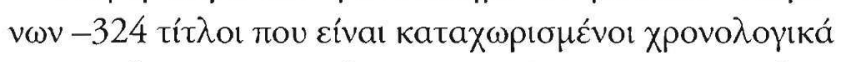

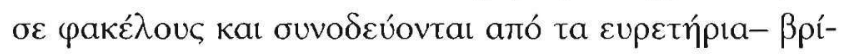

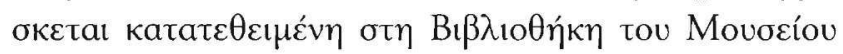

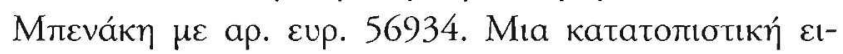

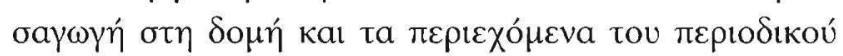

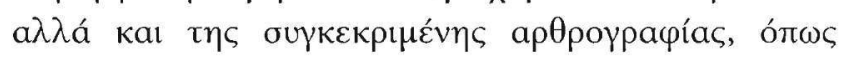

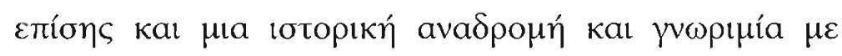

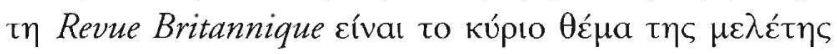
autท́c.

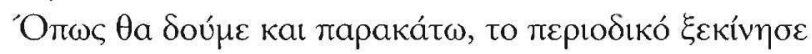

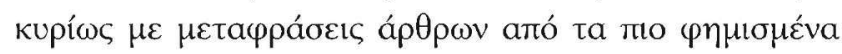

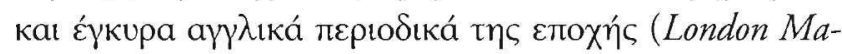
gazine, Literary Gazette, New Monthly Magazine, Edin-

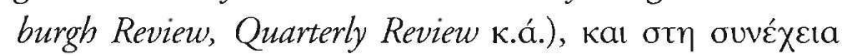

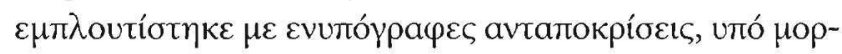

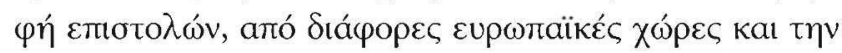

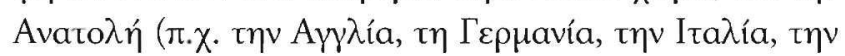

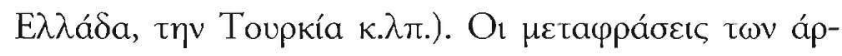

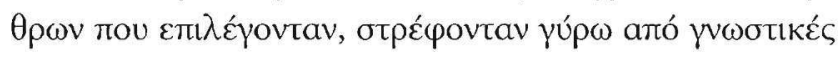

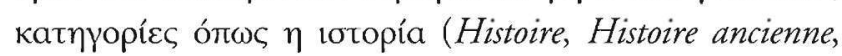

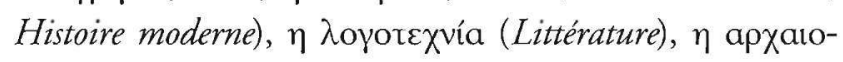

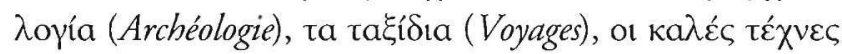

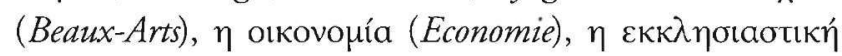

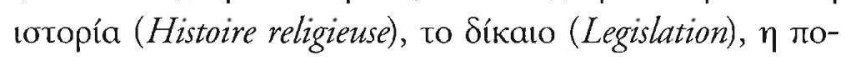
$\lambda_{\iota \tau \iota \kappa}$ (Politique, Politique etrangère, Politique extérieure),

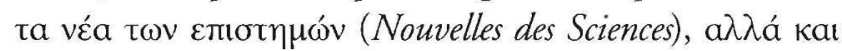

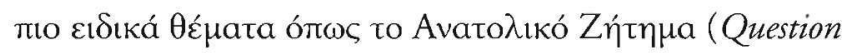

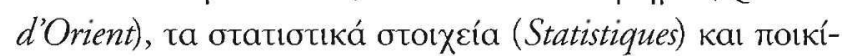
$\lambda \varepsilon \varsigma$ á $\lambda \lambda \varepsilon \varsigma \mu 1 \kappa \rho \varepsilon ́ \varsigma \pi \lambda \eta \rho о \varphi о \rho i ́ \varepsilon \varsigma$ (Miscellanées).

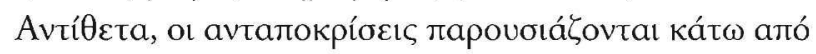

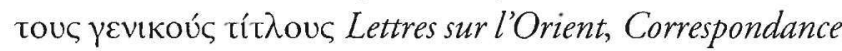
d'Orient, Correspondance et chroniques.

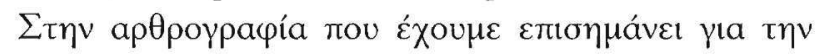

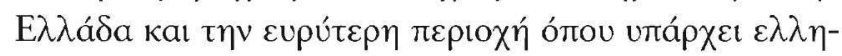

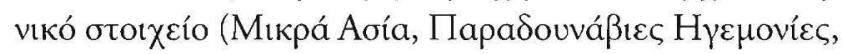

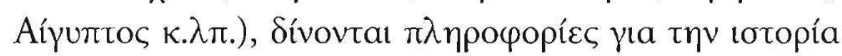

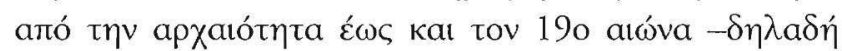




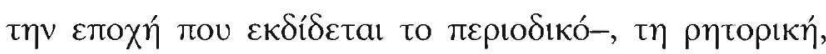

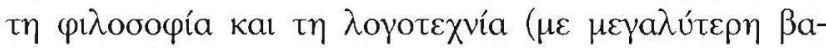

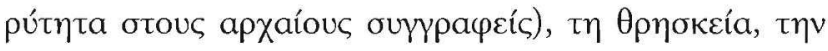

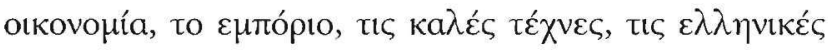

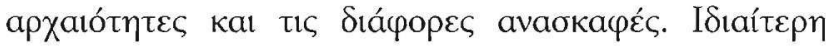

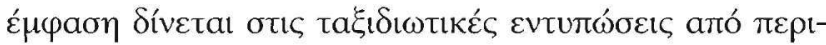

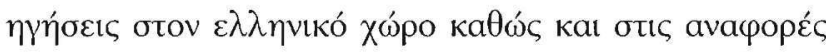

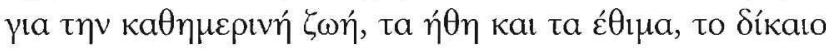

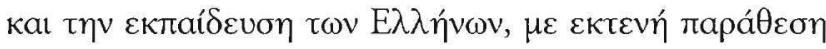

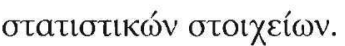

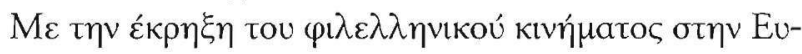


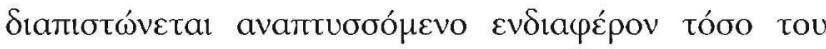

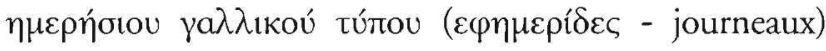

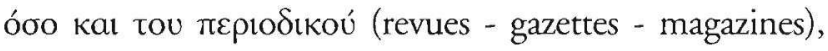

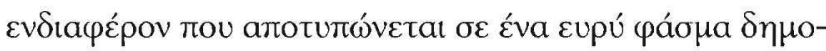

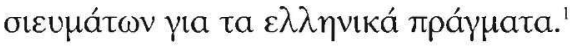

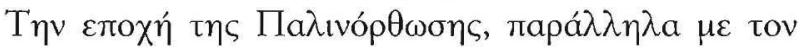

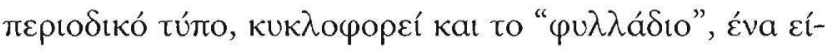

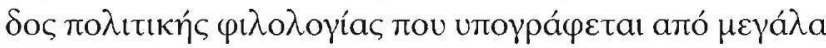

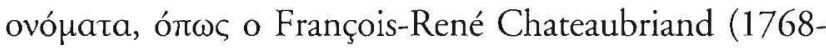
1848), o Felicité-Robert de La Mennais (1782-1854),

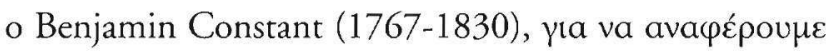

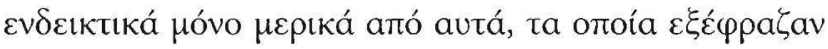

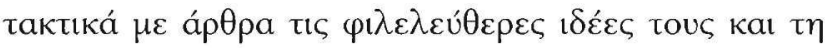

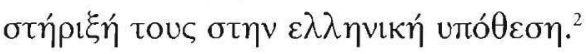

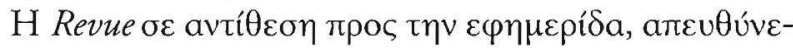

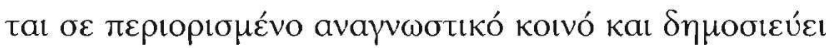

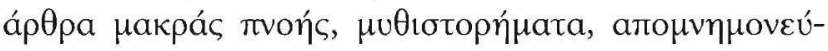

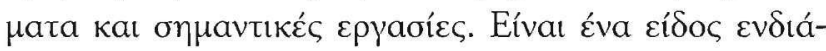

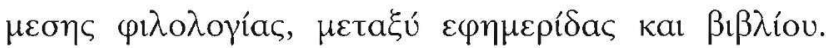

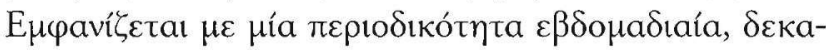

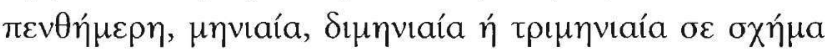

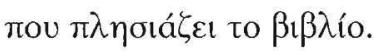

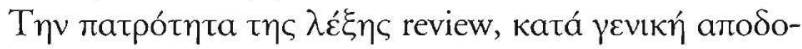

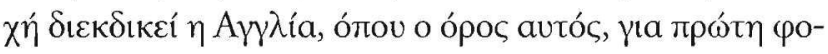

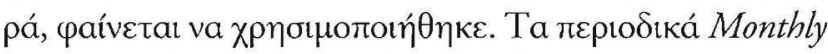

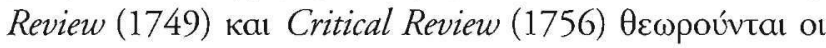

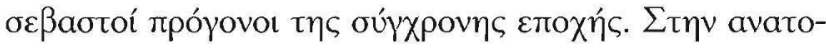

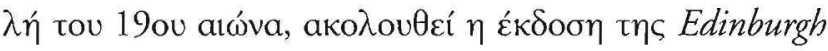
Review (1802), tou Quarterly Review (1809), tov Foreign Quarterly Review (1824), tou Westminster Review (1827) кaı tou New Quarterly Review (1852). Ta терıобıќ́

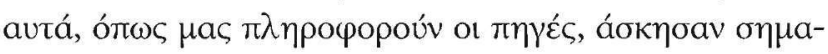

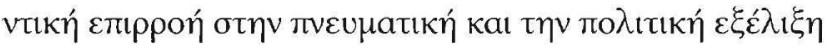

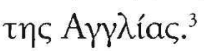

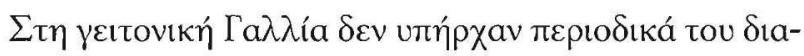

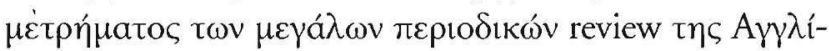

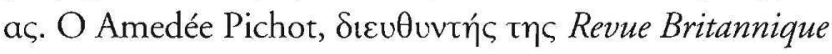

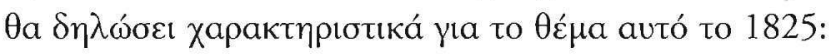

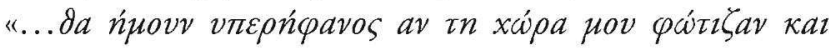

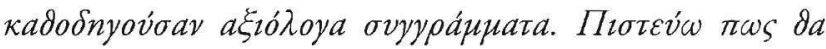

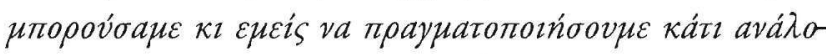

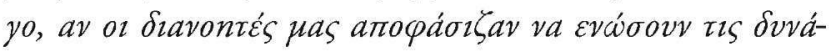

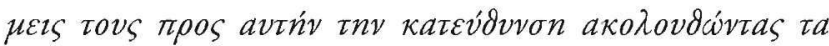

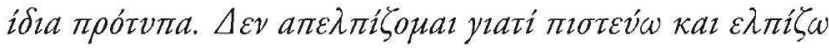

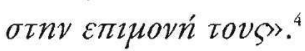

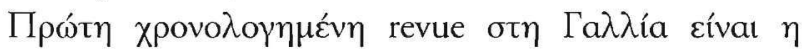

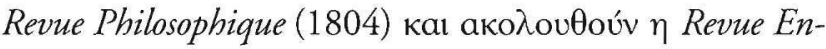
cyclopédique (1818), $\eta$ Revue Britannique (1825), $\eta$ Revue de Paris (1829) кaı $\eta$ Revue des deux Mondes (1829) к.á.

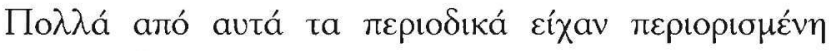

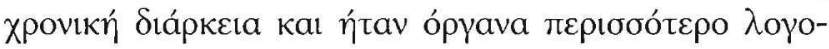

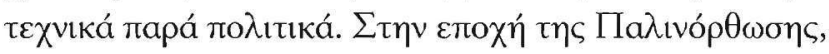

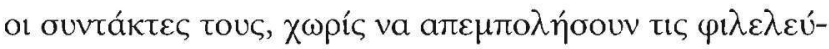
$\theta \varepsilon \rho \varepsilon \varsigma$ по入

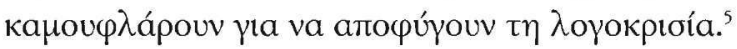

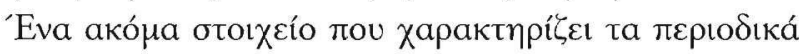

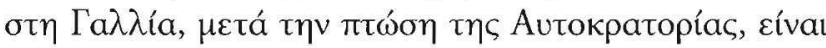

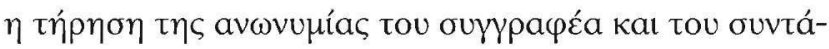

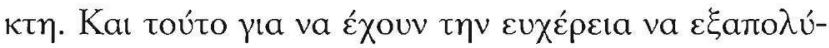

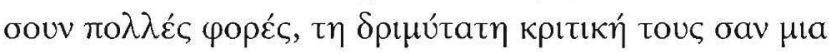

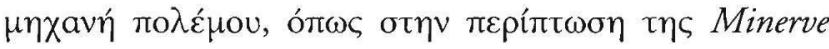
Française, tou Conservative, tou Globe kau tou Figaro. $\Sigma \varepsilon$

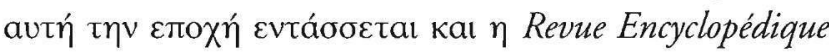

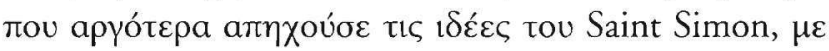

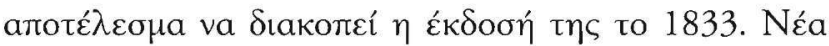

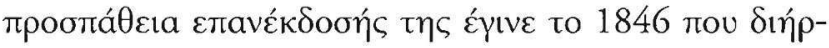

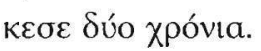

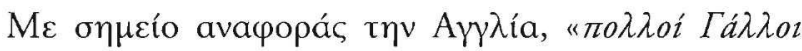

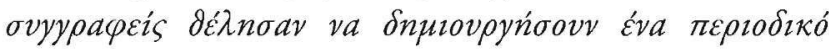

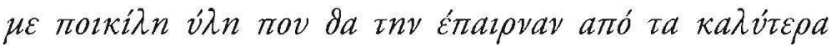

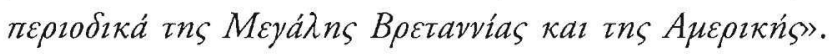

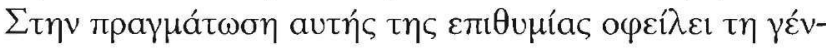

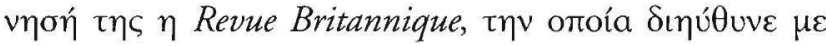

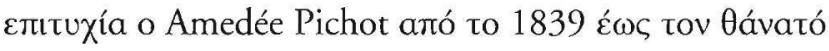
tov to $1877 .{ }^{\circ}$

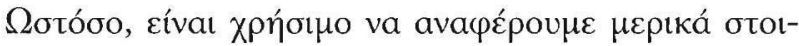

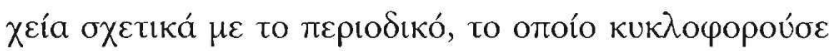

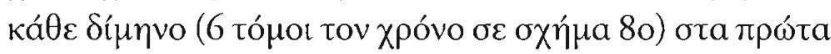

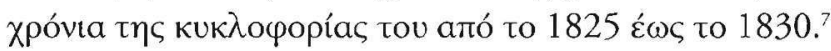

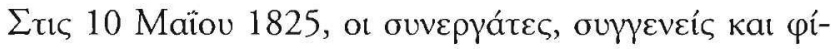


$\lambda o t$, Sebastien-Louis Saulnier, Jean-Michel Berton кaı

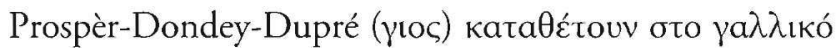

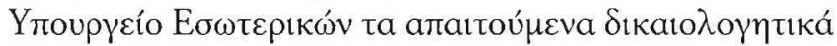

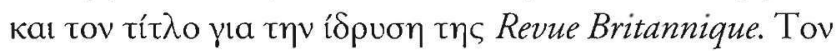

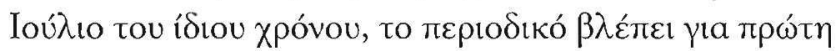

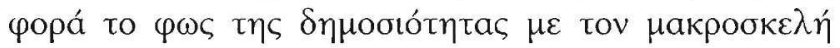
tít入o: Revue Britannique, ou choix d'articles traduits des meilleurs écrits périodiques de la Grande-Bretagne, sur la littérature, les beaux-arts, les arts industriels, l'agriculture, le commerce, l'économie politique, Les finances, la législation, etc., etc., Par MM. Charles Coquerel; Dondey-Dupré fils, de la Société Asiatique; Gerusez; Ed. Lafon de Labebat, ancien chef de division au Ministère de l'Interieur; Raulin, des Sociétés Asiatique et de Géographie; Saulnier fils, ancien Préfet de la Société Asiatique; West Docteur en Médecine (pour les articles relatifs aux sciences médicales) etc., etc., Paris 1825.

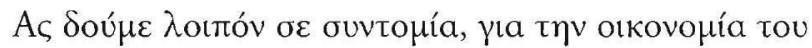

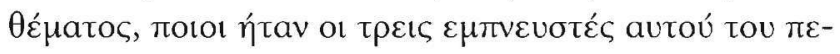

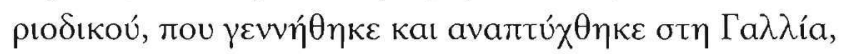

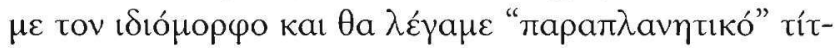

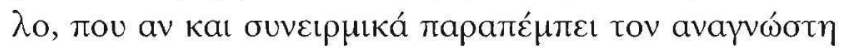

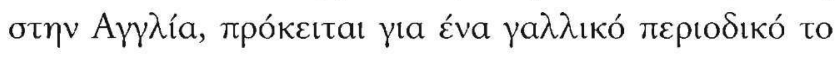

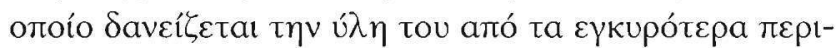

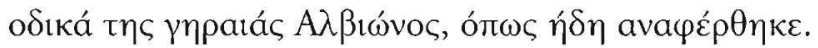

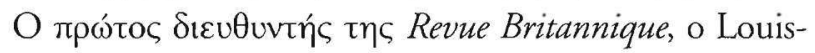

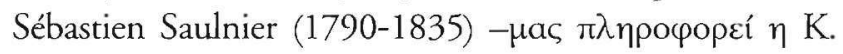

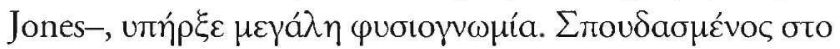

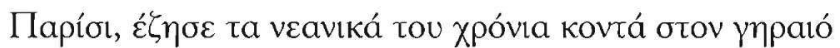

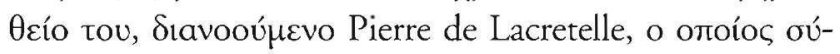

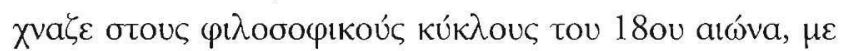

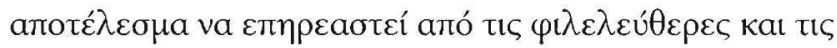

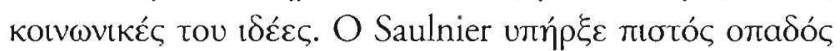

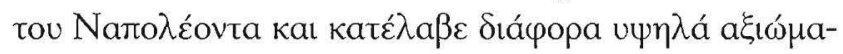

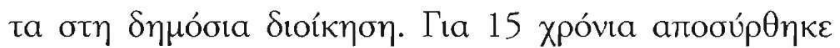

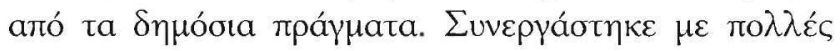

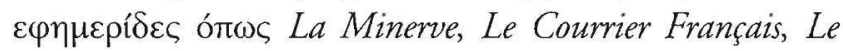

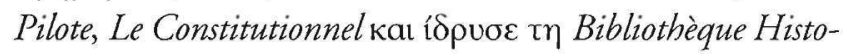

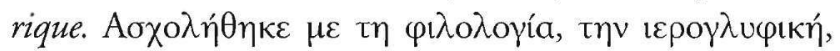

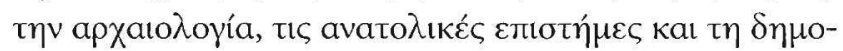

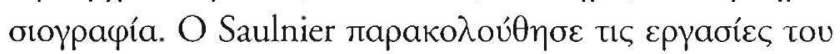

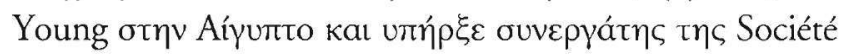

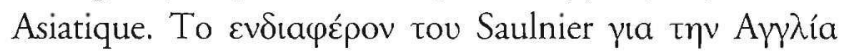

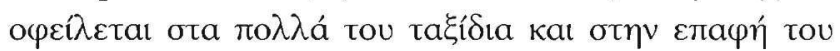

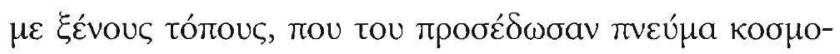

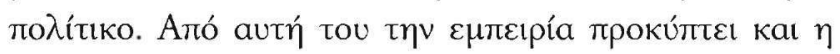

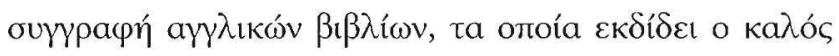

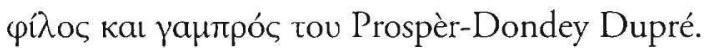

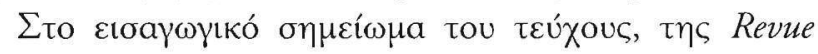
Britannique tou Iavovapíou 1829, o Saulnier $\gamma$ pápel:

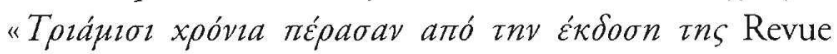

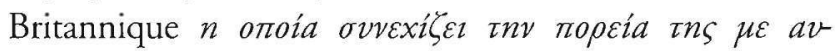

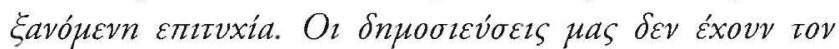

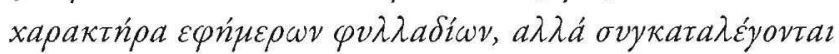

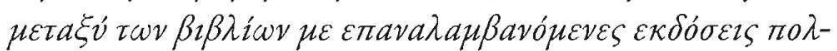

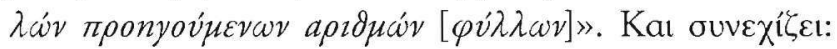

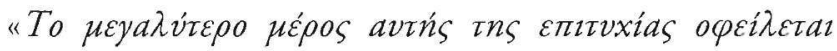

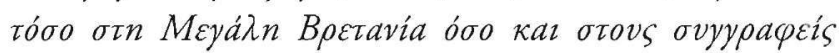

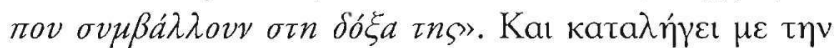

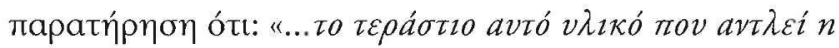

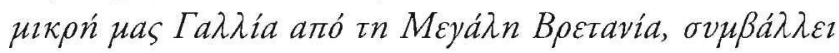

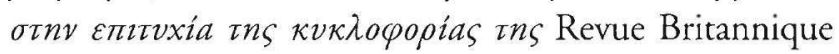

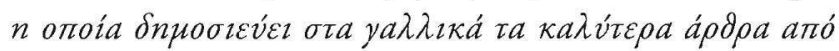

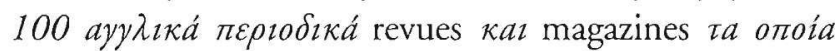

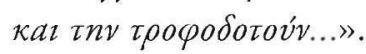

O Jean-Michel Berton (1793-;) ota 30 tou xpóvia

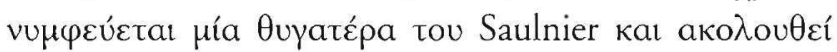

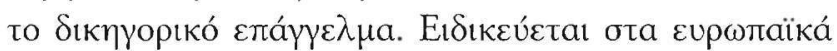

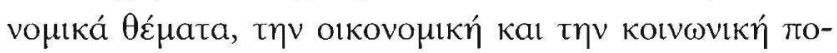

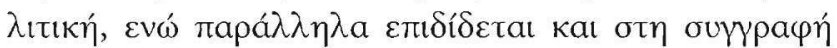

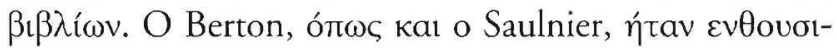

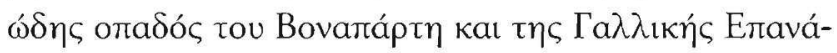

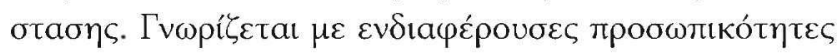

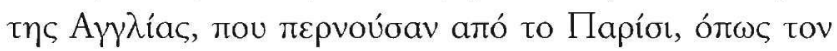
B. P. Shelley, tov Wordsworth kat rov Charles Coc-

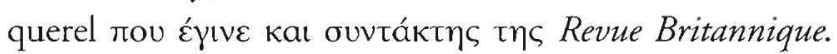

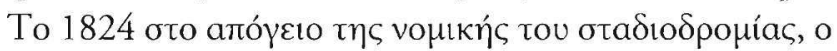

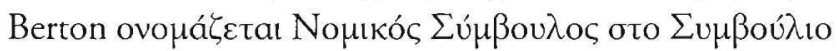

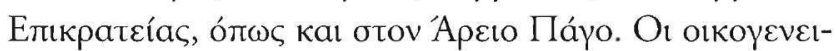

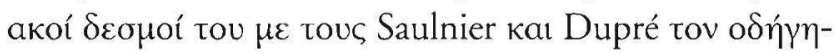

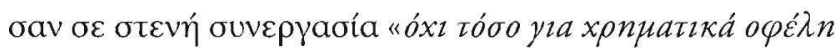

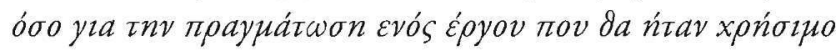

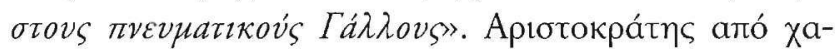

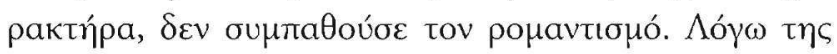

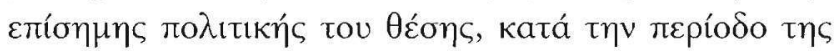

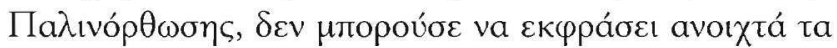

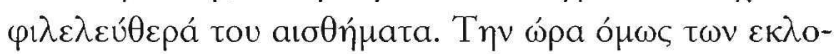

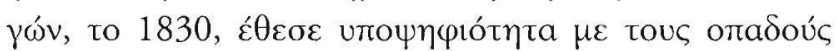

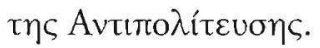

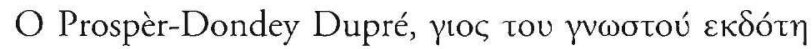

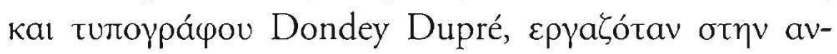

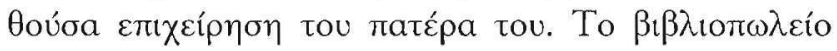




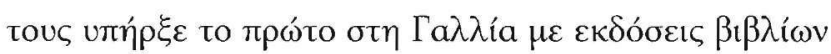

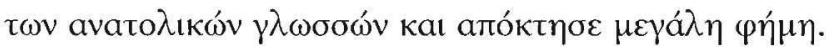

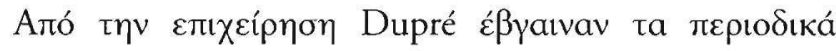
Revue Asiatique, Revue Protestante ka Revue Germanique.

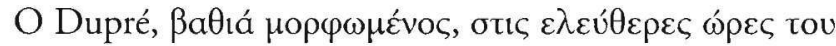

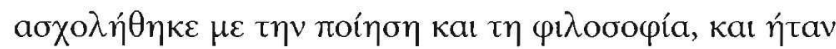

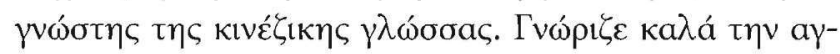

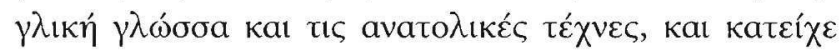

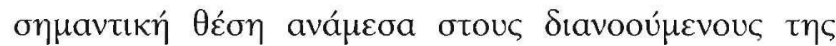

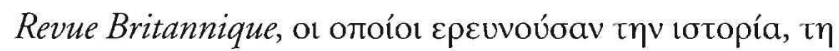

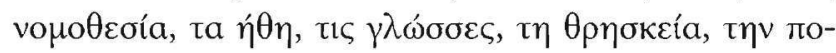

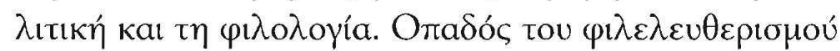
-ó

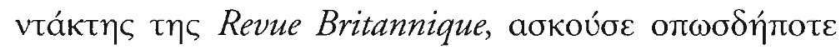

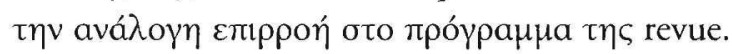

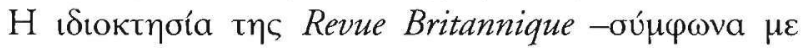

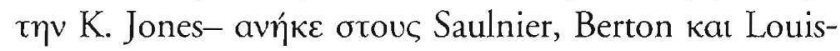

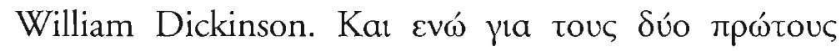

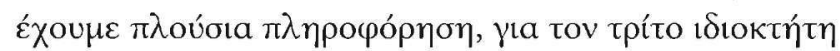

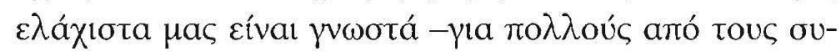

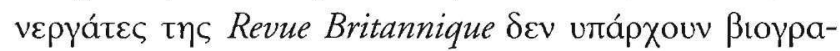

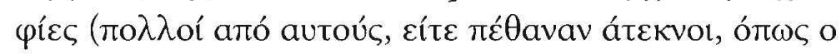

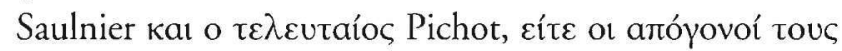

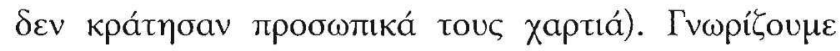

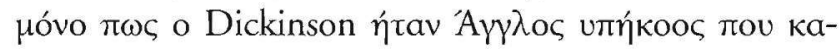

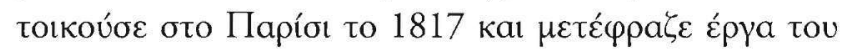

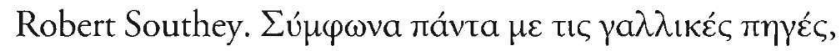

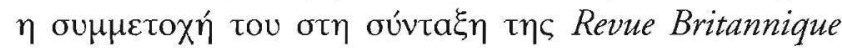

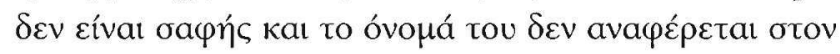

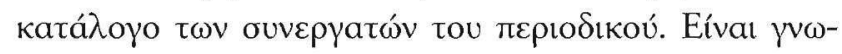

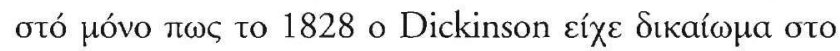

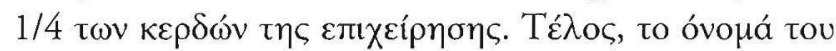

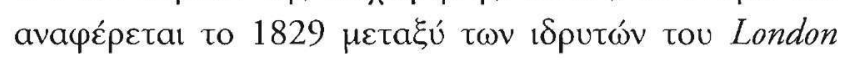

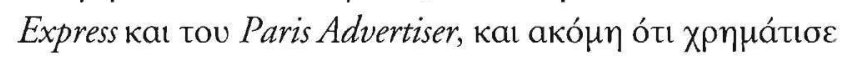

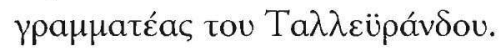

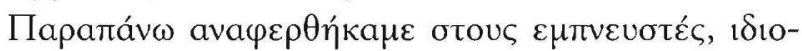

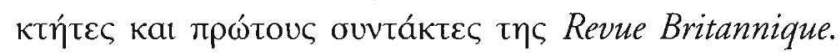

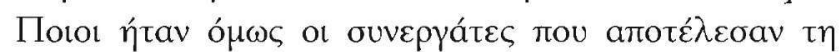

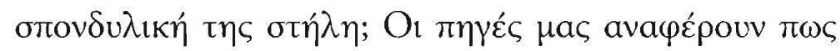

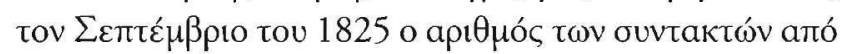

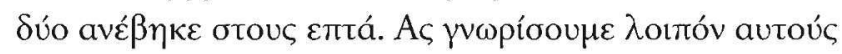

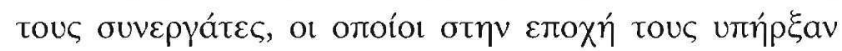

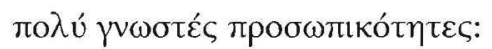

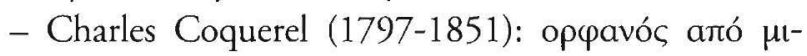

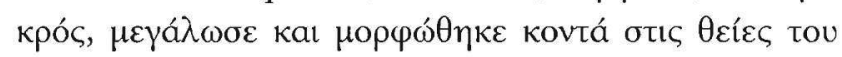

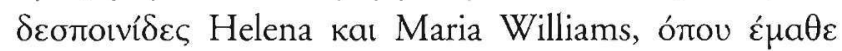

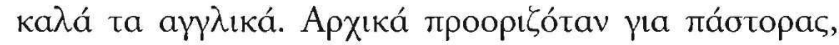

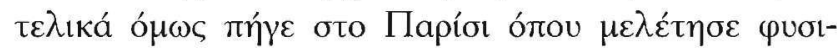

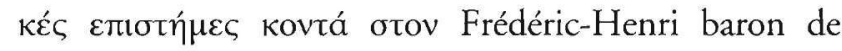
Humboldt (1769-1859), таракодоúӨ

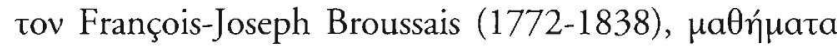
apxaı̀oүíaৎ aтó tov Jean-Antoine Letronne (1787-

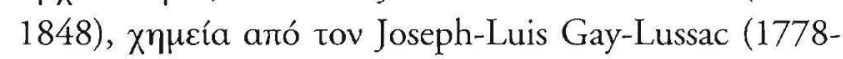

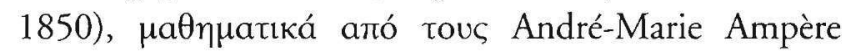
(1775-1836) кal Jean-Baptiste Biot (1774-1862), каı aotpovouía aлó tov Dominic-François Arago (1786-

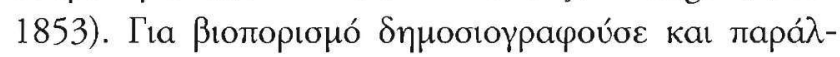

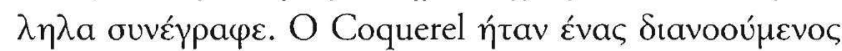

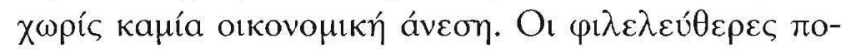

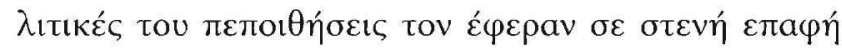
$\mu \varepsilon$ tov Marie-Jean-Paul Lafayette (1757-1834) кal tov Benjamin Constant (1767-1830).

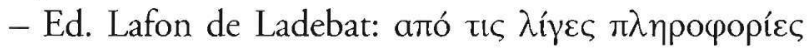

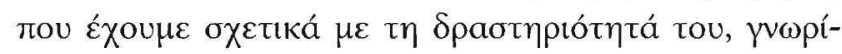

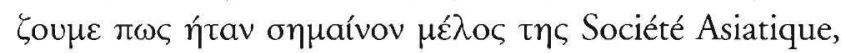

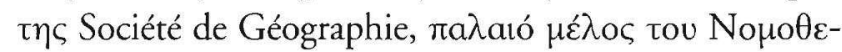

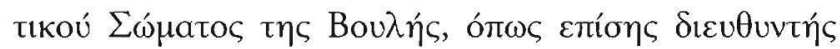

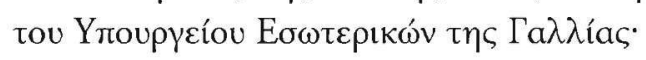

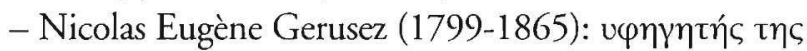

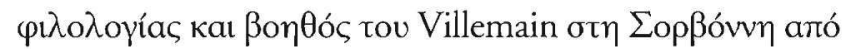

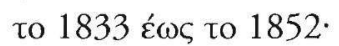

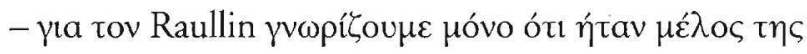
Société Asiatique kal тฑৎ Société de Géographie.

- to ísı kal yı tov West, о олоíos ท́tav yıatpóc.

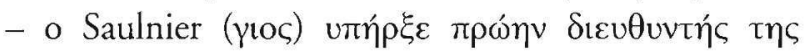
Société Asiatique.

- o Jérôme-Adolphe Blanqui (1798-1854), Évac aró

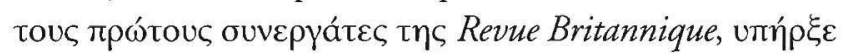

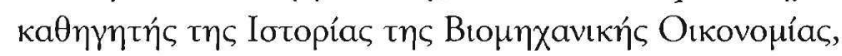

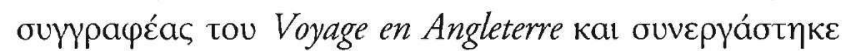

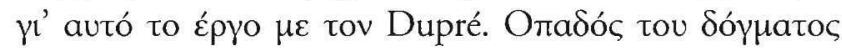

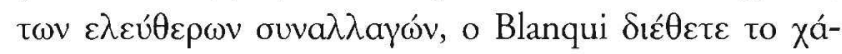

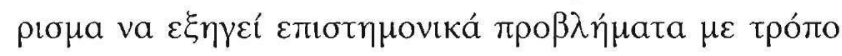

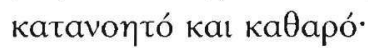

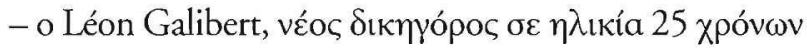

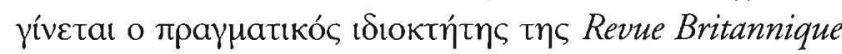

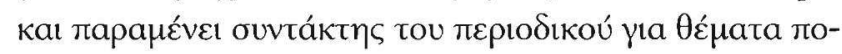

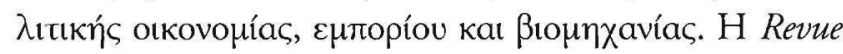

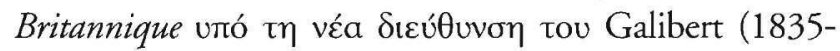

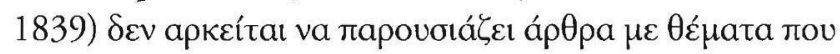

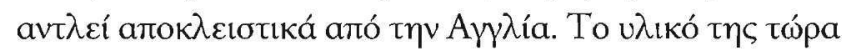

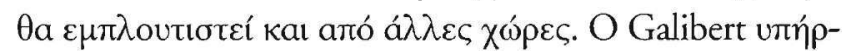




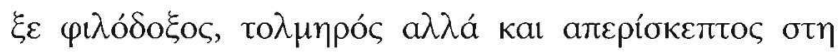

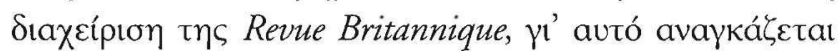

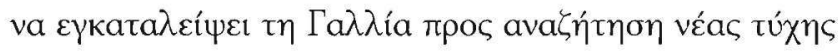

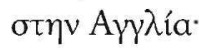

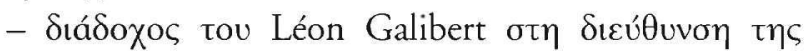

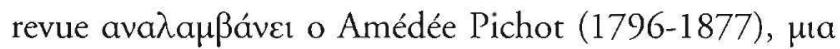

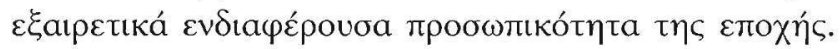

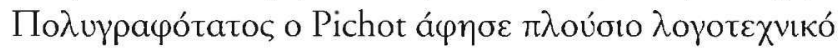

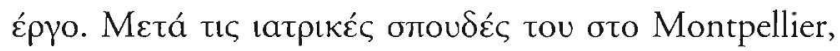

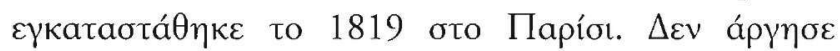

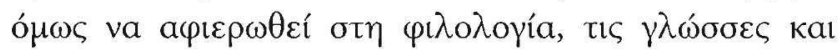

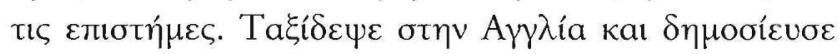

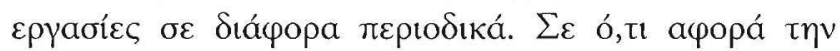

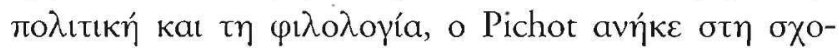

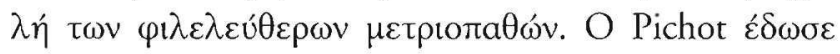

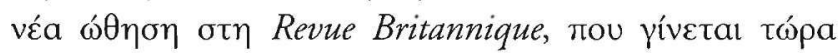

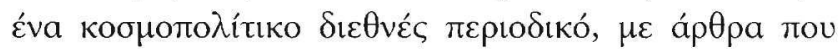

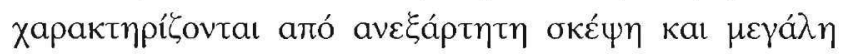

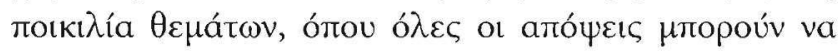

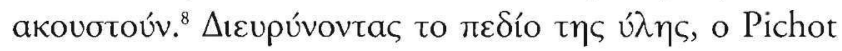

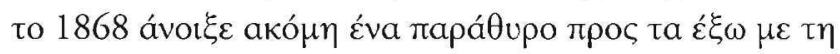

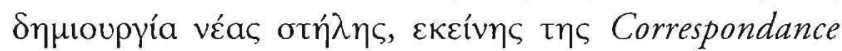

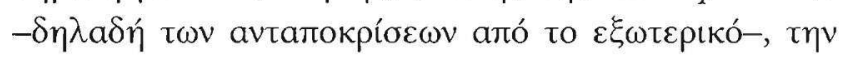

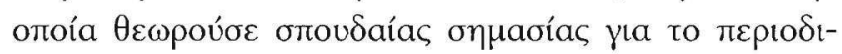

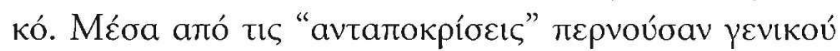

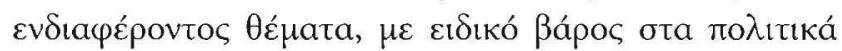

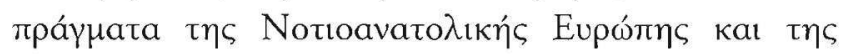

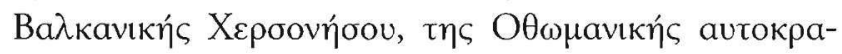

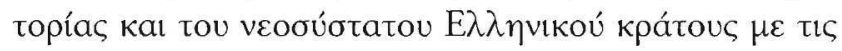

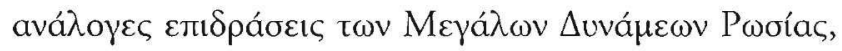

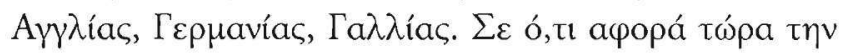

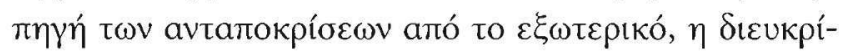

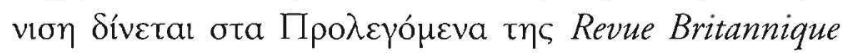

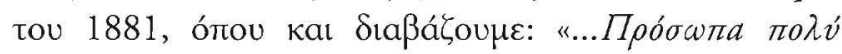

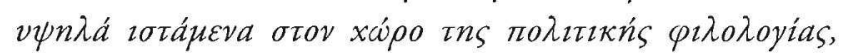

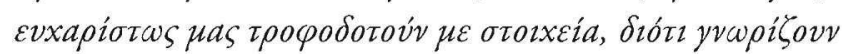

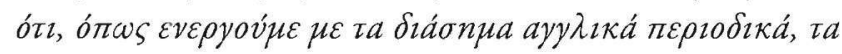

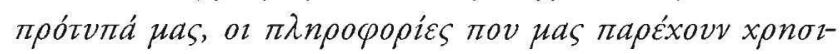

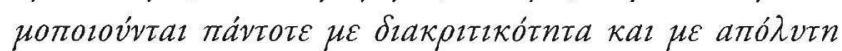

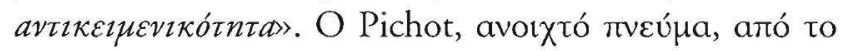

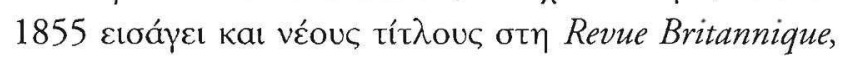
ó $\omega \varsigma$ ta Chroniques littéraires et bulletins bibliographiques

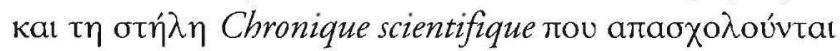

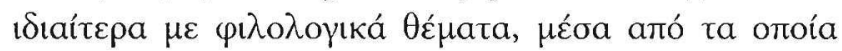

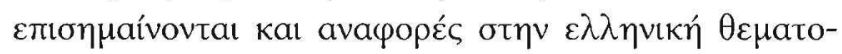
үрачía.

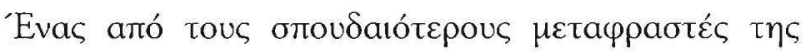

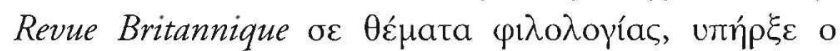

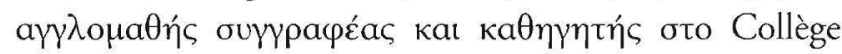
de France, o Philarète Charles (1798-1873), о олоíos

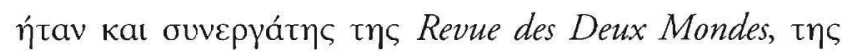

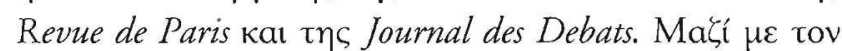

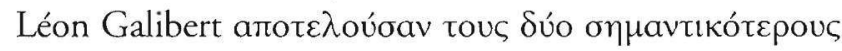

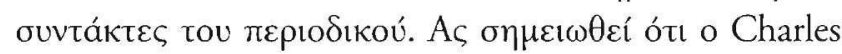

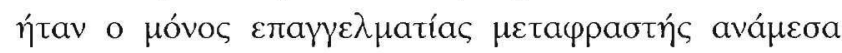

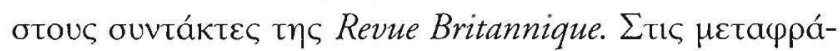

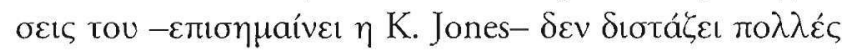

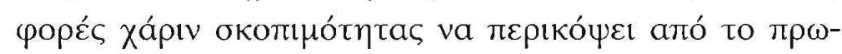

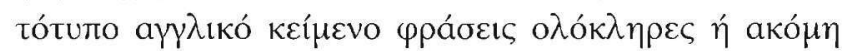

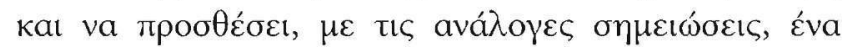

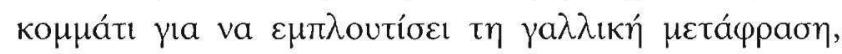

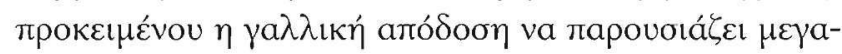

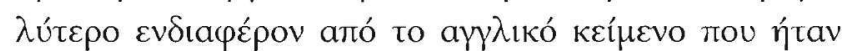

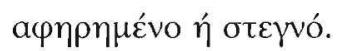

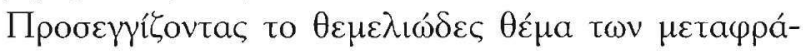

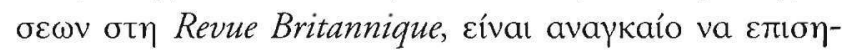

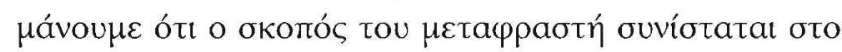

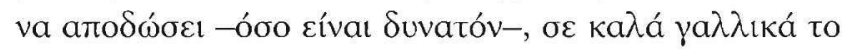

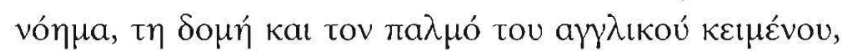

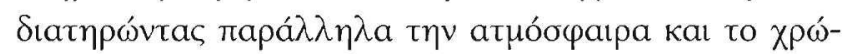
$\mu$ a tov. A A $\lambda \lambda \omega \sigma \tau \varepsilon, \eta$ Revue Britannique cíval śva ópyavo

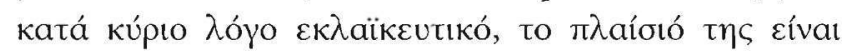

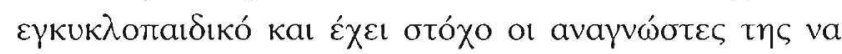

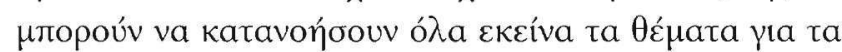

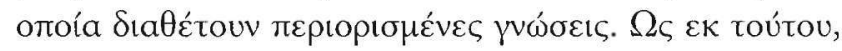

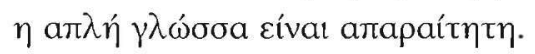

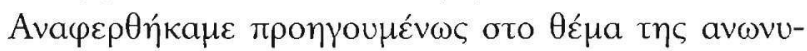

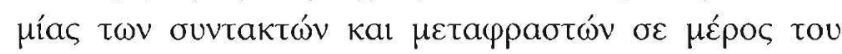

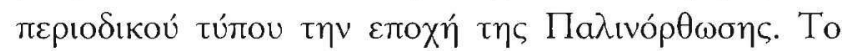

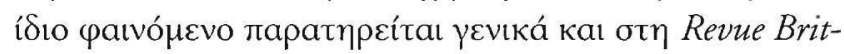

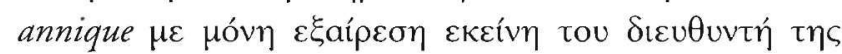

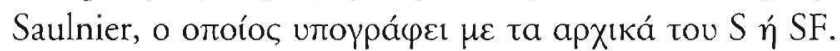
Aкó $\mu \eta$ ú́a popá aravtá to CC (Charles Coquerel) oro

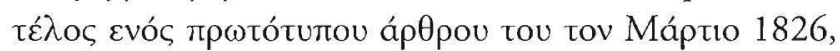

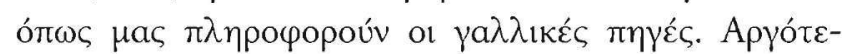

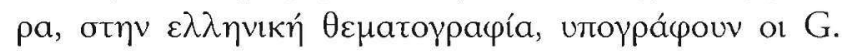
d'Orcet, Pierre Guerraz, Octave Sachot, Amedée Pichot кaı Pierre A. Pichot.

Metá tov Gávato tov Amedée Pichot to 1877, кaı түv

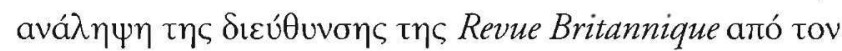

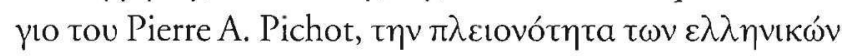

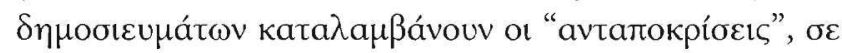




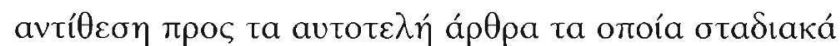

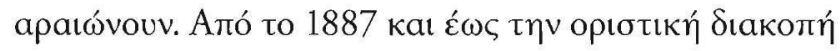

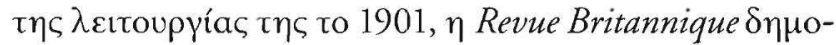

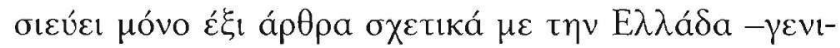

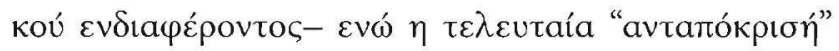

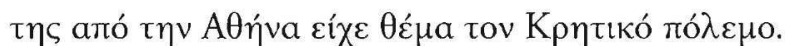

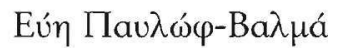

Eovndias 69-71

11521 Adńva

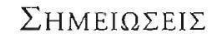

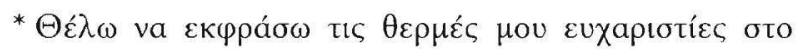

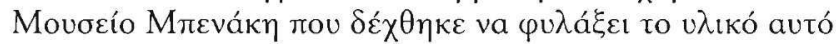

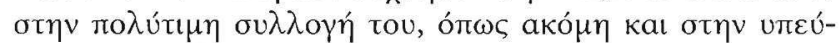

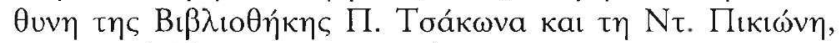

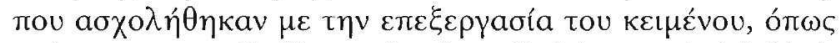

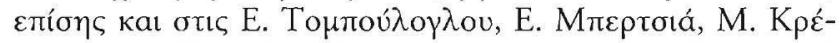

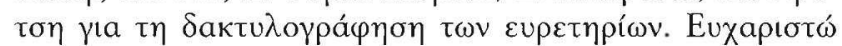

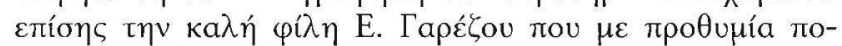

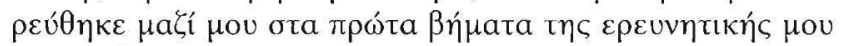

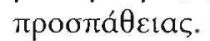

1. A. Dimopoulos, L'opinion publique française et la revo-

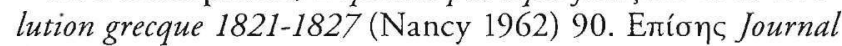
de Delé-cluze 1824-1828 (Paris 1948) 345. A. Варvaкı́́-

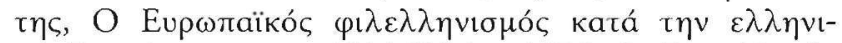

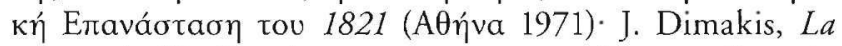
guerre de l'indépendance grecque vue par la presse française

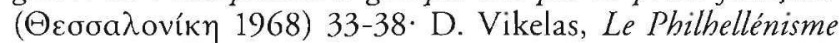
en France (extrait de la Revue d'Histoire diplomatique [Paris 1891] 15-16).

2. J. Dimakis, La société de la Morale Chretienne de Paris et son action en faveur des Grecs lors de l'insurrection de 1821,

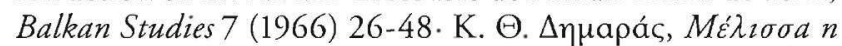

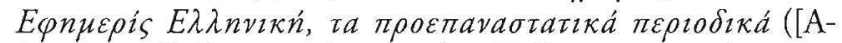

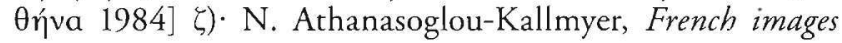

from the Greek War of Independence 1821-1830 (New HavenLondon 1989) 8-13.

3. G. Vapereau, Dictionnaire Universel des litteratures (Paris 1876) 1723-25.

4. A. Pichot, Voyage pittoresque et littéraire en Anglettere et en Ecosse. Ladvocat et Charles Gosselin 2 (Paris 1825) 253. J. Dimakis, La presse française face à la chute de Missolonghi et à la bataille navale de Navarin; recherches sur les sources de

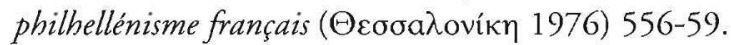

5. Dimakis (ó.л.) K. Jones, La Revue Britannique, son histoire et son action littéraire (1825-1840) (Paris 1939) 23-24.

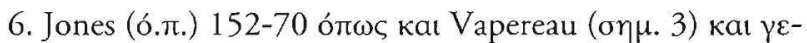
vıкótepa, L.-E. Hatin, Histoire de la Presse en France (Paris 1861) vol. 8: Revues, 609-15.

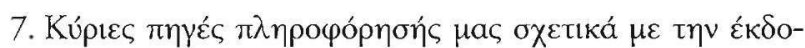

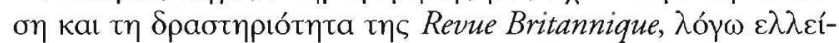

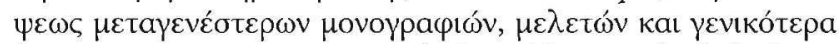

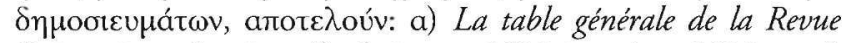
Britannique depuis sa fondation en 1825 jusqu' en 1880 par J. Drapier (publiée sous la direction de Pierre Amedée Pichot, 1881,

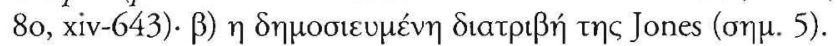

8. Revue Britannique, 1881, Avant propos, v-ix.

\section{EVI PAVLOV-VALMA}

Revue Britannique and its Greek material

The Benaki Museum library has recently acquired a series of 324 photocopied items from the celebrated French periodical Revue Britannique, which circulated in Paris between 1825 and 1901. These are independent articles and brief reports on matters of general interest relating to Greece, its history and its culture, from antiquity until the 19th century. The significance of this material, collected in photocopied form after research in Swiss libraries, lies in the fact that the magazine is not to be found in any library in Greece -apart from an incomplete set in the National Library. The material has been catalogued and arranged in chronological order; indexes by name and subject have been prepared for use by future researchers. 
The texts give special emphasis to travel writing and impressions by visitors to Greece and its wider area, antiquities, everyday life, manners and customs, religion and the Greek legal and educational systems, with extensive use of statistical information. This study ends with a general history of Revue Britannique, which was first published at the outbreak of the philhellenic movement in Europe and for many years played a major role in the French press, always taking an interest in issues related to Greece. 